\title{
Investigation of a steady-state cylindrical magnetron discharge for plasma immersion treatment
}

\author{
I. Levchenko a) and M. Romanov \\ Kharkov National Aerospace University, Kharkov, Ukraine \\ M. Keidar ${ }^{\text {b) }}$ \\ Department of Aerospace Engineering, The University of Michigan, Ann Arbor, Michigan 48109
}

(Received 28 February 2003; accepted 14 May 2003)

\begin{abstract}
Ion current distribution in a system with crossed magnetic and electrical fields for plasma immersion ion implantation has been investigated. It is found that the ion current to a target has a nonmonotonic behavior with bias voltage when a magnetic field is applied. For instance, the current density has a maximum of about $150 \mathrm{~A} / \mathrm{m}^{2}$ at bias voltage of about $1 \mathrm{kV}$ in the case of a magnetic field parallel to the target of about $0.035 \mathrm{~T}$. These results are explained in terms of ionization by magnetized electrons in the $E \times B$ system. Our findings suggest that the system with crossed fields can be used for intense plasma immersed processing. (C) 2003 American Institute of Physics.
\end{abstract}

[DOI: $10.1063 / 1.1590054]$

\section{INTRODUCTION}

Plasma immersion technique is used, along with conventional methods, for modification of surface properties of metals and semiconductors. ${ }^{1,2}$ The main advantage of this technique, as compared with the conventional line-of-sight ion beam technology, consists in a possibility to process complex-shaped targets. In this process the target is immersed into a plasma generated in a vacuum chamber. Negative bias voltage is applied to the target, this causes bombardment of the target surface by ions extracted from the plasma and accelerated in sheath area between the target surface and plasma. The plasma immersion process at high voltage was found to be successful for treatment of different materials. ${ }^{3-5}$ Along with high-voltage process, the relatively low-voltage treatment is also effective..$^{6,7}$

The processes that occur near the target surface (in the plasma sheath) during extraction of ion flow from the plasma are most important for surface treatment. Some effects were studied previously for systems without magnetic field ${ }^{8,9}$ and in the presence of transverse magnetic field ${ }^{10}$ for the case of voltage applied to the target in pulses with a frequency of several Hz. It was also shown recently that magnetic field can be used for control of the sheath expansion. ${ }^{10}$ In this article we present the results of experimental investigations of processes in the system with dc magnetic and electrical fields applied.

Usage of the steady state electrical and magnetic fields during the implantation process provides several advantages. Application of steady voltage does not require high-voltage interrupters and high-voltage pulse generators. ${ }^{11,12}$ It is known that the arc discharges between the chamber walls and the target often take place during a voltage rise phase ${ }^{13}$ and deteriorate the target surface quality. ${ }^{14}$ Besides, simulta-

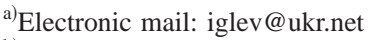

b) Author to whom correspondence should be addressed; electronic mail: keidar@engin.umich.edu
}

neous coating deposition and immersion treatment provides new possibilities for a film structure improvement due to surface heating, coating impregnation with doping materials, and activation of adsorbed atoms on the substrate surface. ${ }^{15}$

\section{EXPERIMENTAL SETUP}

A scheme of experimental setup is shown in Fig. 1. In our experiment we used an axial system with permanent magnets similar to that used in cylindrical magnetrons. ${ }^{16}$ This scheme ensures magnetic confinement of electrons around the target, in contrast to the electrostatic confinement. ${ }^{17}$ This system is somewhat similar to one used in Hall thrusters ${ }^{18}$ in which ionization by magnetized electrons drifting in the crossed $E \times B$ fields takes place. We did not use the special anode, so the grounded vacuum chamber walls were under the positive potential relative to the target. A cylindrical target (ion current collecting electrode) of 200 $\mathrm{mm}$ length and $72 \mathrm{~mm}$ diameter made of polished nonmagnetic stainless steel was placed into the axial magnetic field created by a set of permanent magnets that can be moved along the longitudinal axis. This way the main component of magnetic field is parallel to the cylindrical target surface above the set of permanent magnets, and intersects the metal surface at the target edges. With target biased, the crossed system of radial electrical field and longitudinal magnetic field is created, providing electron drift around the target. Using different quantity of magnets with different magnetization we ensured creation of various strengths of magnetic fields with the same configuration. The screen and upper lid were installed on the ceramic rings and isolated from the target, so they were placed under the floating plasma potential with a view to prevent arcing on the target edges. The polished heat screen made of nonmagnetic stainless steel was placed between the cylindrical target and magnets to decrease radiant heating (see Fig. 1).

In these experiments a water-cooled cylindrical vacuum chamber with a volume of $0.25 \mathrm{~m}^{3}$ (inner diameter $0.55 \mathrm{~m}$ 


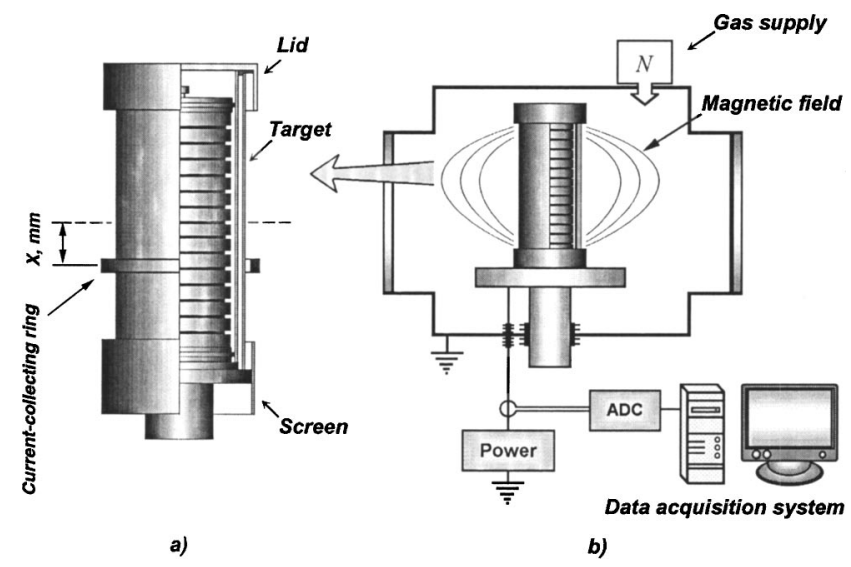

FIG. 1. Magnetic unit (a) and experimental setup (b).

and length $1.0 \mathrm{~m}$ ) was used. The chamber was equipped with two hinged doors and two windows. The vacuum system incorporates one mechanical backing pump and one highvacuum oil pump with maximum pumping rate of $1500 \mathrm{l} / \mathrm{s}$. The automatic gas-supplying system maintained nitrogen pressure in a range of $0.1-10 \mathrm{~Pa}$. The pressure was maintained by controlling the rate of nitrogen inflow using the electromagnetic valve.

The pressure was measured with a help of the thermocouple vacuum gage and two ionization gages. One ionization gage was used as a probe for the gas-supplying system, and one gage was used for the data registration. The current, voltage and pressure were registered by automated data acquisition system (DAS) connected to a computer via the analog-digital converter. The DAS was provided with a highvoltage decoupler capable of withstanding dc voltages up to $10 \mathrm{kV}$. The power supply system used in this setup was able to provide voltage variation from 0 to $2 \mathrm{kV}$ at maximum current up to $20 \mathrm{~A}$. The assembled magnetic unit [see Fig. 1(a)] was installed on negatively biased rotating holder located in the geometrical center of the vacuum chamber. The

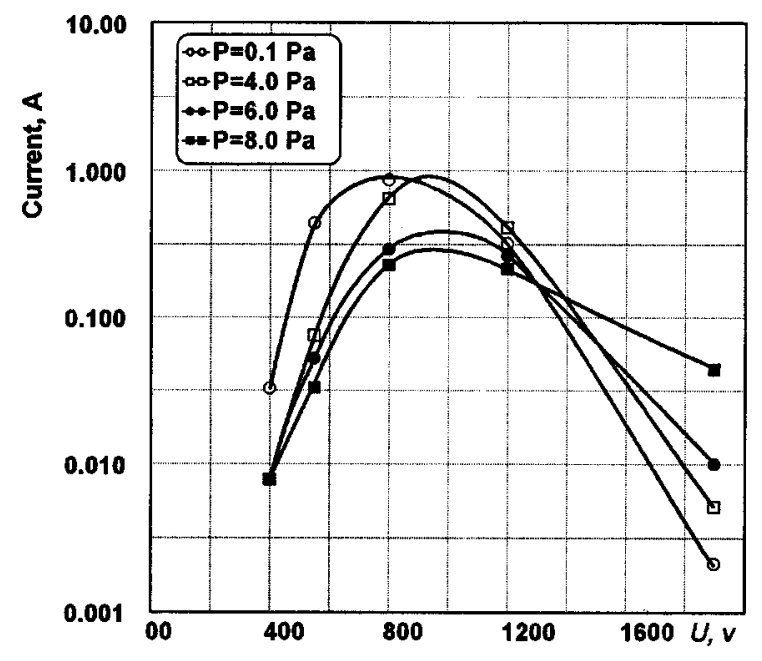

a current distribution along the target surface was measured with a narrow ( $3 \mathrm{~mm}$ wide) current-collecting ring installed on the target and isolated from the target surface with a hightemperature insulator. The ring was connected to the power supply via separate ammeter.

\section{RESULTS}

Figures 2(a) and 2(b) illustrate the total currents to the target surface as a function of voltage with pressure as a parameter. It can be seen from Fig. 2(a) that the total current has a peak of about $1 \mathrm{~A}$ when the magnetic field of $0.035 \mathrm{~T}$ is applied. A similar experiment without the magnetic field has demonstrated that the current does not exceed $0.01 \mathrm{~A}$ [Fig. 2(b)]. Taking into account that the current was collected mainly by the circular area with a width of $2-3 \mathrm{~cm}$ in magnetic field and by the entire target surface without the field (as evident from the hot electrode and plasma glow), we obtain the current density peak of about $150 \mathrm{~A} / \mathrm{m}^{2}$ in magnetic field and current density to the target surface of about $0.5 \mathrm{~A} / \mathrm{m}^{2}$ without the magnetic field.

When a magnetic field is applied, the total currents to the target surface as a function of voltage has strongly pronounced maximum at voltages of about $800-1000 \mathrm{~V}$ as shown in Fig. 2(a). Besides, the arcing is absent at such conditions, whereas it was impossible to reach a voltage of 2 $\mathrm{kV}$ at zero magnetic field due to very frequent arc breakdowns between the biased target and grounded chamber walls.

The total currents to the target surface as a function of pressure with bias voltage and magnetic field as parameters are shown in Fig. 3. One can see that the current-pressure dependencies have also a complex nonmonotonic behavior. The current decreases with pressure increasing for low voltages (curves for 600, 800, and $1200 \mathrm{~V}$ ), and increases for a voltage exceeding $1350 \mathrm{~V}$ (curves for 1600 and $1800 \mathrm{~V}$ ). The same effect can be noticed also in Fig. 2(a) (the currentvoltage curves intersect in the point of about $1350 \mathrm{~V})$. With-

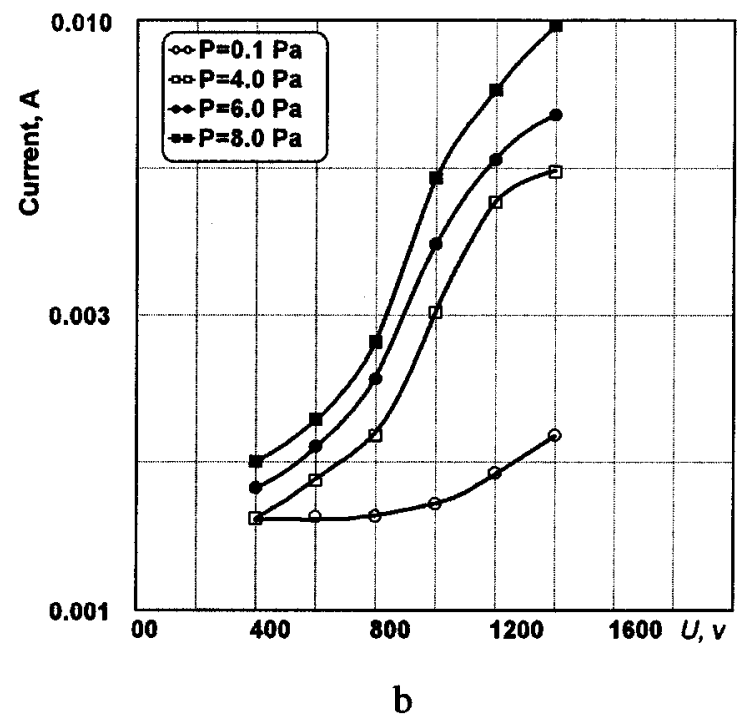

FIG. 2. Current-voltage curves with pressure as a parameter: (a) $B=0.035 \mathrm{~T}$ and (b) $B=0$. 


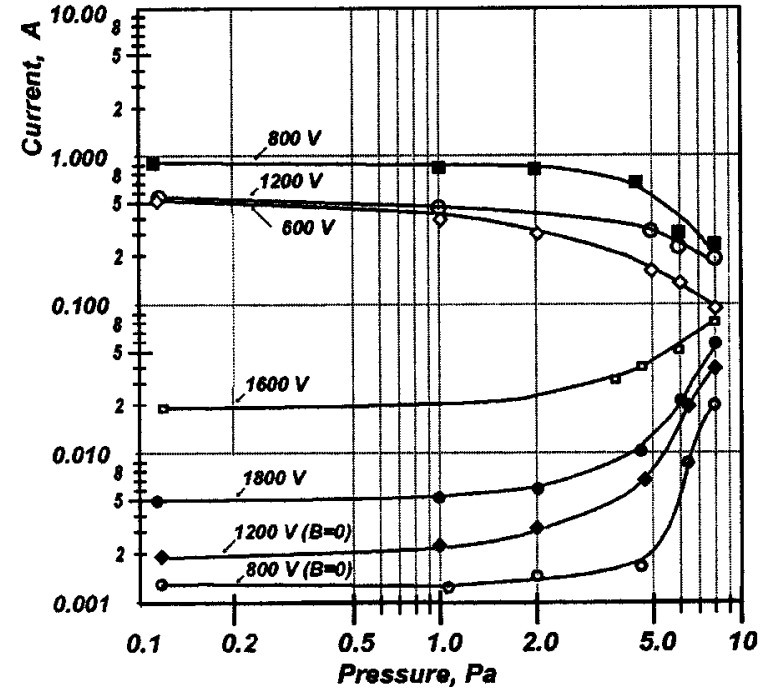

FIG. 3. Current-pressure curves with voltage and magnetic field as parameters.

out magnetic field, the current always increases with the pressure increase (two lower curves in Fig. 3).

The dense plasma areas around the magnetic unit for magnetic field strength of $0.035 \mathrm{~T}$ and different voltages are shown in Fig. 4. It can be seen that at a voltage of $1000 \mathrm{~V}$ the plasma has the form of a well-shaped torus with a diameter of several centimeters [see Fig. 4(a)]. The plasma glow is very bright, and the dominant color is bluish-pink. The ion current in this case is collected mainly on the narrow circular

$1 \mathrm{~cm}$
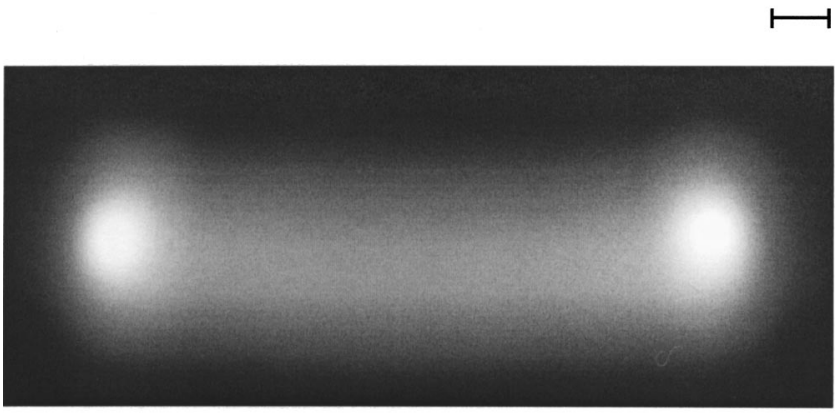

a)

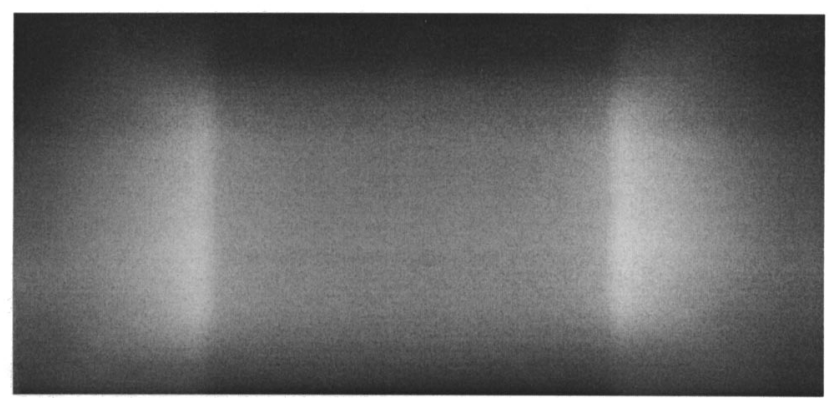

b)

FIG. 4. Photographs of plasma around the magnetic unit. High current (a), $B=0.035 \mathrm{~T}, U=1000 \mathrm{~V}$. Low current (b), $B=0.035 \mathrm{~T}, U=1600 \mathrm{~V}$. area under the plasma torus, with a width of about $3 \mathrm{~cm}$. The dense plasma area probably corresponds to the closed electron drift region. ${ }^{18}$ This target area can be brought to white heat for several seconds. The process becomes unstable when magnetic field increases above $0.05 \mathrm{~T}$, and the following current pulsations accompanied by plasma flashes lead to power unit overload, which was the cause for termination of the experiment. It can be mentioned that the plasma is ignited gradually for several seconds, but not instantly after applying a voltage corresponding to the strong current.

With a voltage increasing over $1000 \mathrm{~V}$, the torus becomes dark, and the current decreases. At a voltage of 1600 $\mathrm{V}$ the plasma glow becomes much dimmer, the well-shaped torus disappears, and the target surface can be seen through the plasma as a dark cylindrical object [see Fig. 4(b)]. The further voltage increase leads to subsequent darkening of the plasma glow, and the total current decreases to several milliamperes or several tens of milliamperes, depending on the pressure.

\section{DISCUSSION}

The configuration used in these experiments is very close to the cylindrical magnetrons, as it was already mentioned in Sec. I. One important distinguishing feature is that we use the target as the part being treated, and do not use the separate substrates located separately, as it is used for magnetron deposition. Actually we do not apply any additional electrodes, so the electric field is created between the target and chamber, separated by a distance of approximately 0.5 $\mathrm{m}$. In this configuration the electron runaway from the discharge area is significantly decreased. Together with the appreciably increased gas pressure, it provides a very intense self-sustaining ionization and very high target current. Comparing the operating parameters of this system and Hall thruster one can conclude that both configurations are close with respect to the main parameters.

The distinguishing feature of this system, as compared with the Hall thruster, is the presence of an ion collector (target). This forces us to take into account the current of secondary electrons from the target surface produced by the ion impact emission. In this case the total current $J$ will be found as

$$
J=\left(1+k_{s i}\right) \cdot J_{i},
$$

where $J_{i}$ is the ion current and $k_{s i}$ is the ion-electron impact emission coefficient. Estimating the ion current to the target one should consider the electron emission and subtract the electron current from the total target current value. It should be noted that the electron emission coefficient does not exceed 0.1 in our conditions (steel target, nitrogen ions with energy up to $1.5 \mathrm{kV}),{ }^{19}$ and hence the ion current is about $90 \%$ of the total current.

We can explain qualitatively observed phenomenon by examining the processes in a partly magnetized plasma near the metal wall. In the Ref. 9 similar reasoning was used for explaining the sheath evolution in the expanding vacuum arc plasma. In our case we deal with a fundamentally steady state plasma without injecting an ion flow from any sources. 


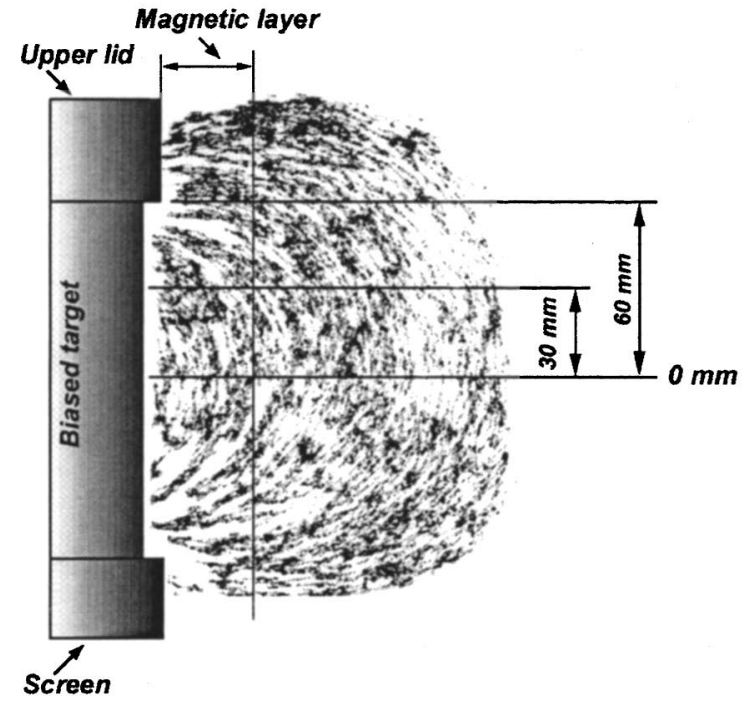

a

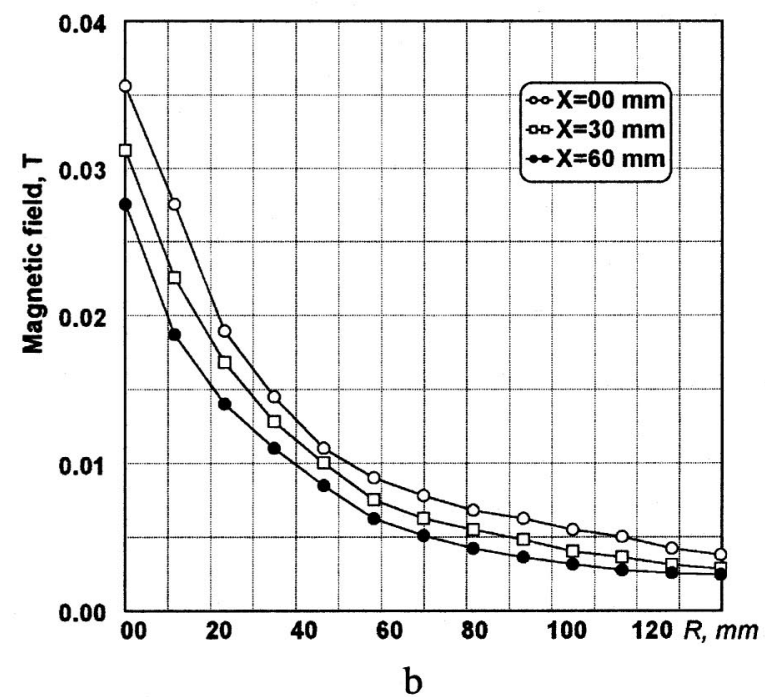

FIG. 5. Magnetic field near target surface: (a) magnetic field topography and (b) dependence of magnetic field on distance from target for different cross sections; $X=0 \mathrm{~mm}$ : plane of symmetry; $X=30 \mathrm{~mm}$ : between center and border; and $X=60 \mathrm{~mm}$ : border.

Therefore we shall examine the equilibrium conditions for the sheath between the partially magnetized plasma and the target.

In steady state the Child-Langmuir equation ${ }^{20}$ for the ion current in the presence of space charge can be used

$$
j_{i}=\frac{4 \epsilon_{0}}{9 \delta^{2}} U^{3 / 2} \sqrt{\frac{2 e}{m_{i}}},
$$

where $j_{i}$ is the ion current density, $U$ is the voltage, $\epsilon_{0}$ is the permittivity of vacuum, $m_{i}$ is the ion mass, and $\delta$ is the sheath thickness. We can calculate the magnitude of the $\delta$ using the measured current density. For our conditions we estimated that the sheath thickness is less than $1 \mathrm{~cm}$ for low voltage and about several centimeters for high voltage. From the above estimations and examination of the magnetic field geometry the following scenario can be proposed. The high current (low voltage) situation corresponds to the case when steady state sheath is much thinner than the magnetic layer (defined as a region with strong magnetic field) outlined by magnetic lines emanating from the target surface near the floating potential lid and screen [i.e., the boundary magnetic lines intersecting the biased electrode, see Fig. 5(a)]. In contrast, the low current (high voltage) situation corresponds to the case when the sheath encloses the magnetic layer.

Equation (2) was derived for the infinite parallel electrodes in the absence of a magnetic field. Strictly speaking, one cannot use this relation for analyzing the processes in a curvilinear system with a magnetic field without special justification. In Ref. 10 this equation was successfully used for analyzing the processes in a spherical system immersed into the symmetric magnetic field. To justify the use of this equation in our conditions, we evaluated the effect of nonlinear geometry and the magnetic field. Comparing the main radius of a plasma torus (about $100 \mathrm{~mm}$ ) and the characteristic thickness of the sheath $(10-35 \mathrm{~mm})$ one can conclude that the errors caused by the nonplanar geometry do not exceed
$10 \%-35 \%$. There is no doubt that those values are quite allowable for our evaluative estimations being used for principal explanation of the observed phenomena. Similar reasoning can be used with respect to the finite size of the electrodes; comparing the electrode size with the sheath thickness we receive an error of about $10 \%-20 \%$.

To evaluate the influence of a magnetic field, let us estimate the maximum and minimum ion Larmor radii in the conditions typical for the sheath

$$
r_{l}=\frac{m_{i} V_{i}}{e B}
$$

where $m_{i}$ is the ion mass, $V_{i}$ is the ion velocity, $e$ is the electron charge and $B$ is the magnetic field strength. Assuming the maximum and minimum bias voltages of 1600 and $400 \mathrm{~V}$, and the magnetic field in the sheath of $0.025 \mathrm{~T}$, we shall have $r_{l \cdot \max }=1 \mathrm{~m}$ and $r_{l \cdot \min }=0.4 \mathrm{~m}$, correspondingly. These values are substantially larger than the sheath thickness, and hence we can consider the ion flow as nondisturbed by the magnetic field.

To check the above assumption on the leading role of the sheath thickness to the magnetic layer width ratio, we measured the plasma density and electron temperature near the target surface, and studied the magnetic field topography. The plasma density and electron temperature were measured with several Langmuir probes for both characteristic conditions, namely for the high current $(U=1000 \mathrm{~V})$ and low current $(U=2000 \mathrm{~V})$ conditions at a pressure of about $4.5 \mathrm{~Pa}$. The measurements showed the density of about $10^{18} \mathrm{~m}^{-3}$ for high-current conditions (in the bright plasma torus) and $10^{16} \mathrm{~m}^{-3}$ for low-current conditions. The electron temperature near the target surface reaches $4.5 \pm 0.5 \mathrm{eV}$ for highcurrent conditions and $5.5 \pm 0.5 \mathrm{eV}$ for low-current conditions. Taking this into account one can readily estimate that the sheath thickness is about several $\mathrm{mm}$ in the high-current case and about $40 \mathrm{~mm}$ in the low-current case. 


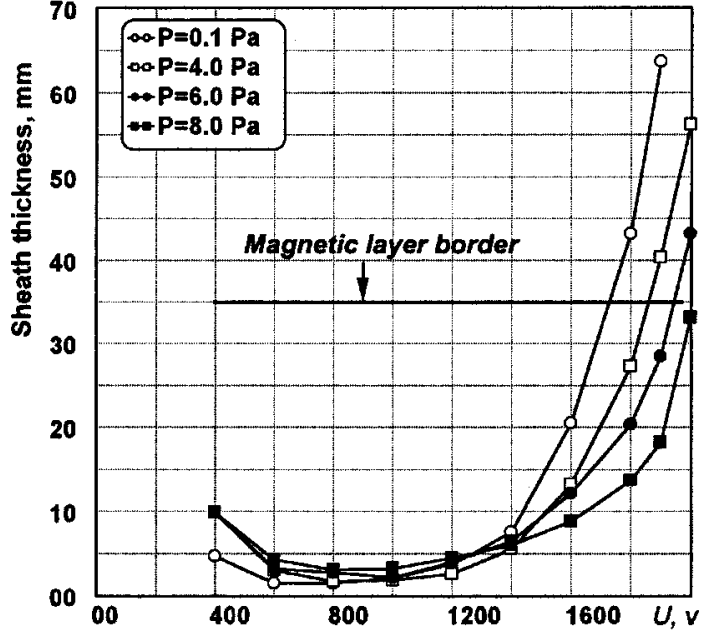

FIG. 6. Dependence of sheath thickness on voltage with pressure as a parameter.

To elucidate the geometry of a magnetic layer and study the influence of a magnetic layer width on the system behavior, we visualized the magnetic field in the near-target area [the topography is shown in Fig. 5(a)] and measured the dependence of a magnetic field on the distance from target surface [Fig. 5(b)]. It can be seen from this figure that the magnetic layer thickness is about $35 \mathrm{~mm}$. Comparing this with our estimations of sheath thickness we can conclude that under low-current conditions the sheath thickness is close to the magnetic layer thickness. In Fig. 6 we show the dependence of the sheath thickness on voltage calculated from Eq. (2). In these calculations we used the measured plasma density. From this figure one can see that the sheath thickness increases significantly when voltage exceeds 1600 $\mathrm{V}$, and approaches (or even exceeds for several pressure values) the magnetic layer width at a voltage of $1800 \mathrm{~V}$. It should be noted that the case of the minimum sheath thickness (from 800 to $1000 \mathrm{~V}$ ) corresponds to the largest drift area; this provides the most intense ionization and therefore largest ion current as shown in Fig. 2(a). Based on these estimations, we can conclude that the behavior of the system considered with crossed electrical and magnetic fields is controlled by the sheath thickness to the magnetic layer thickness ratio.

The visual observations of this process showed that the target surface in high-current conditions is heated mainly in the center area located symmetrically relative to the upper and lower screens, i.e., under the plasma torus. We measured the current distribution on the target surface with the help of a narrow current-collecting ring installed on the target and capable of moving along the magnetic unit axis. The measurements for two pressure values show (see Fig. 7) that the current to the target surface has a significant maximum under the plasma torus. In contrast, no significant nonuniformity was found for the low-current condition (lower curve at Fig. 7). In this case the current to target surface is distributed uniformly, like the plasma glow shown in Fig. 4(b). The current distribution under the plasma torus can be regarded as relatively uniform within the circular area of $2.5-3 \mathrm{~cm}$.

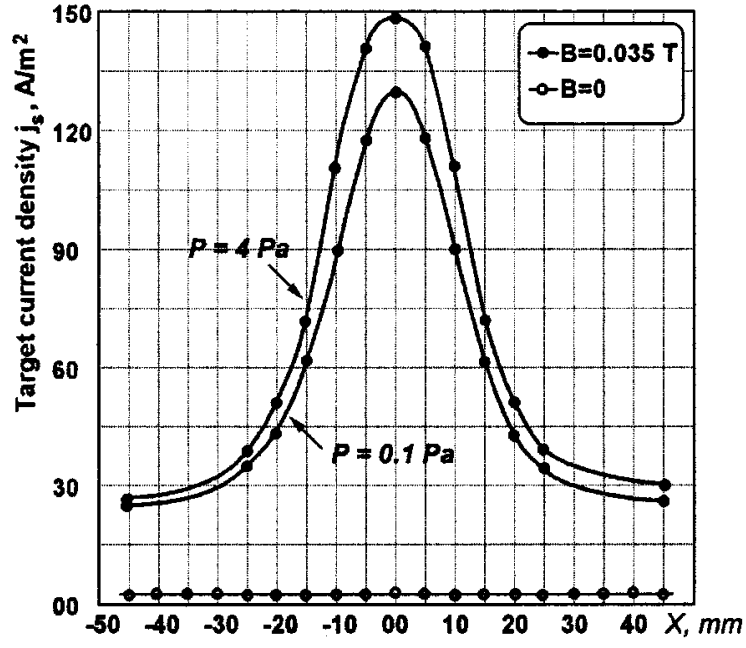

FIG. 7. Current distribution on target surface with pressure and magnetic field as parameters: $U=1000 \mathrm{~V}$.

Examining this phenomena with respect to the practical applications, we can note that the intense uniform treatment of large areas can be provided for by use of several methods, such as enlarging the magnetic field area or moving the crossed-field area with electromagnetic coils.

In the system considered, the ion current to the target is provided mainly by electron impact ionization of the reactive gas. To examine the conditions of electron retention in the cross-field area, let us write the electron current in crossed electrical and magnetic fields in the classical form:

$$
j_{e}=e \cdot n_{e} \cdot \boldsymbol{\mu}_{e} \cdot \mathbf{E}
$$

where $n_{e}$ is the electron density, $\boldsymbol{\mu}_{e}$ is the electron mobility, and $\mathbf{E}$ is the electrical field. The electron mobility tensor may be written in the following form: ${ }^{21}$

$$
\boldsymbol{\mu}_{e}=\left[\begin{array}{ccc}
0 & \mu_{\mathrm{ed}} & 0 \\
0 & \mu_{e t} & 0 \\
0 & 0 & \mu_{p}
\end{array}\right],
$$

where $\mu_{p}$ is the electron mobility along the magnetic field, $\mu_{\text {ed }}$ is the electron drift mobility, and $\mu_{e t}$ is the electron cross-field mobility. Assuming the classical form of the electron mobility, we have

$$
\mu_{p}=\frac{e}{m_{e} \nu_{e}}, \quad \mu_{\mathrm{ed}}=\mu_{p} \frac{\varpi}{1+\varpi^{2}}, \quad \mu_{e t}=\mu_{p} \frac{1}{1+\varpi^{2}},
$$

where $\varpi=e B / \nu_{e} m_{e}$ is the electron Hall parameter, and $\nu_{e}$ is the electron-atom collision frequency. For our cross-field system we have only drift $\left(j_{\text {ed }}\right)$ and cross-field $\left(j_{e t}\right)$ electron currents that can be written as

$$
j_{\mathrm{ed}}=e n_{e} E \mu_{p} \frac{\varpi}{1+\varpi^{2}}, \quad j_{e t}=e n_{e} E \mu_{p} \frac{1}{1+\varpi^{2}},
$$

From Eq. (7) one can see that the ratio of drift current $j_{\text {ed }}$ and cross-field current $j_{e t}$ is equal to $\varpi$ and can be estimated to be about 10-100 for our conditions (assuming the gas pressure of $0.1-8 \mathrm{~Pa}$, electron temperature $5 \mathrm{eV}$, and electronatom collision cross section of $2 \times 10^{-19} \mathrm{~m}^{2}$ ). These esti- 
mates show that the electron drift current well exceeds the cross-field current, and the conditions for intense ionization are provided around the target similar to the Hall thruster. ${ }^{18}$ The electron drift from the ionization area (plasma torus) is small and this provides space charge compensation around the target. As a result, the electrical field is decreased near the target, and the electron mobility is also very low near the surface since the magnetic field has a maximum value in this region.

It must be noted that in our conditions the electron Larmor radius does not exceed $1 \mathrm{~mm}$, and the electrons are well magnetized relative to the sheath thickness (which changes from several $\mathrm{mm}$ to several $\mathrm{cm}$ ), and relative to magnetic layer that is about $30-40 \mathrm{~mm}$. When sheath is thin with respect to the magnetic layer, magnetized electrons are involved in the circular drift motion in the magnetic field outside of the sheath. As a result, the intense ionization takes place in the magnetic layer and high ion flux is provided to the sheath edge and therefore to the target. This situation leads to the well-shaped plasma torus formation, as shown in Fig. 4(a). In the high voltage case, when the magnetic layer is immersed into the expanded sheath (i.e., the sheath encloses the magnetic layer), the conditions are different. Leaving the sheath, electrons simultaneously leave the region of the strong magnetic field and intense ionization cannot be provided. Consequently, the plasma torus is not formed [see Fig. 4(b)], and the ion current to the surface decreases. Thus, in this system the increase of the bias voltage leads to expanding the sheath over the magnetic layer, followed by a sharp decrease of the electron density in the magnetic layer and hence termination of the intense ionization. These effects lead to a very strong decrease of the ion current, as shown in Fig. 2(a).

The same argument enables explanation of the currentpressure behavior shown in Fig. 3. When bias voltage is low and the sheath is much thinner than the magnetic layer, the electron diffusion along the electric field (and across the magnetic field) increases with the pressure increase due to higher electron-atom collision frequency according to the relation

$$
\mu_{e t}=\frac{m_{e} \nu_{e}}{e B^{2}} .
$$

Estimating the classical electron-atom collision frequency as: $\nu_{e}=n_{e} \cdot \sigma_{e} \cdot V_{e}$, where $\sigma_{e}$ is the electron-atom collision cross section, and $V_{e}$ is the electron thermal velocity, we have $\nu_{e} \approx 5 \times 10^{8} \mathrm{~s}^{-1}$ for high pressure conditions and $\nu_{e}$ $\approx 5 \times 10^{6} \mathrm{~s}^{-1}$ for low pressure conditions. Thus one can see that the pressure increase in the experiment described causes an electron mobility increase by 2 orders of magnitude. This causes depletion of the magnetized area and depression of ionization; as a result, the ion current decreases. In the opposite case, i.e., at high voltage, the sheath encloses the magnetic layer. In this case the pressure increase leads to ionization rate growth that in turn causes decrease of the sheath thickness [according to Eq. (1)]. The sheath becomes thinner than the magnetic layer, and the conditions for magnetized electrons accumulation in the magnetic field above the sheath is provided. The ionization in the magnetized area outside of the sheath provides the current rise with the pressure.

In the high-pressure limit, all curves tend to the same current range, i.e., the weak current-voltage dependence is observed (see Fig. 3). In this case the sheath thickness becomes thinner than the magnetic layer for all voltages [due to high pressure, from $3 \mathrm{~mm}$ for $800 \mathrm{~V}$ to $10 \mathrm{~mm}$ for $1800 \mathrm{~V}$, according to Eq. (1)], and the voltage drop being present mainly in the sheath does not affect the current significantly. The ion current is determined mainly by the electron impact ionization in the magnetic layer and electron flux to the sheath, which has only weak dependence on the voltage applied.

\section{SUMMARY}

In summary, we have found that the system with crossed electrical and magnetic fields provides a very high ion current to the target immersed in the plasma. The current density increases by more than 2 orders of magnitude in comparison to the case without magnetic field. The ion current as a function of the bias voltage has a maximum corresponding to $800-1000$ Vin the case of 0.03-0.04 T magnetic field. These results can be explained in terms of ionization by magnetized electrons in the $E \times B$ system. These results suggest that a magnetic field can be used for intensification of the plasma treatment. One possible application of this system is a plasma immersion ion implantation in which ion extraction from the plasma in the steady state regime may be very beneficial.

\footnotetext{
${ }^{1}$ Handbook of Plasma Immersion Ion Implantation and Deposition, edited by A. Anders (Wiley, New York, 2000).

${ }^{2}$ N. W. Cheung, Surf. Coat. Technol. 156, 24 (2002).

${ }^{3}$ A. Loinaz, M. Rinner, F. Alonso, J. I. Onate, and W. Ensinger, Surf. Coat. Technol. 103-104, 262 (1998).

${ }^{4}$ M. Ueda, L. A. Berni, R. M. Castro, A. F. Beloto, E. Abramof, J. O. Rossi, J. J. Barroso, and C. M. Lepienski, Surf. Coat. Technol. 156, 71 (2002).

${ }^{5}$ I. G. Brown, X. Godechot, and K. M. Yu, Appl. Phys. Lett. 58, 1392 (1991).

${ }^{6}$ B. Terreault, Surf. Coat. Technol. 156, 13 (2002).

${ }^{7}$ S. Mukherjee, J. Chakraborty, S. Gupta, P. M. Raole, P. I. John, K. R. M. Rao, and I. Manna, Surf. Coat. Technol. 156, 103 (2002).

${ }^{8}$ M. Keidar, O. R. Monteiro, and I. G. Brown, Appl. Phys. Lett. 76, 3002 (2000).

${ }^{9}$ I. G. Brown, O. R. Monteiro, M. M. M. Bilek, M. Keidar, E. Oks, and A. Vizir, Rev. Sci. Instrum. 71, 1086 (2000).

${ }^{10}$ M. Keidar, O. R. Monteiro, A. Anders, and I. D. Boyd, Appl. Phys. Lett. 81, 1183 (2002).

${ }^{11}$ I. G. Brown, O. R. Monteiro, and M. M. M. Bilek, Appl. Phys. Lett. 74, 2426 (1999)

${ }^{12}$ I. G. Brown, Surf. Coat. Technol. 136, 16 (2001).

${ }^{13}$ D. H. L. Goodall and G. M. McCracken, 19, 1396 (1979).

${ }^{14}$ I. Levchenko, A. Voloshko, M. Keidar, and I. I. Beilis, IEEE Trans. Plasma Sci. 31, 137 (2003).

${ }^{15}$ A. Anders, Surf. Coat. Technol. 93, 158 (1997).

${ }^{16}$ A. Yonesu, H. Takemoto, M. Hirata, and Y. Yamashiro, Vacuum 66, 275 (2002).

${ }^{17}$ A. Metel, Surf. Coat. Technol. 156, 38 (2002).

${ }^{18}$ V. V. Zhurin, H. R. Kaufman, and R. S. Robinson, Plasma Sources Sci. Technol. 8, R1 (1999).

${ }^{19}$ Physical Values, edited by P. Grigor'ev (Nauka, Moscow, 1991) (in Russian).

${ }^{20}$ C. D. Child, Phys. Rev. 32, 492 (1911).

${ }^{21}$ F. F. Chen, Introduction to Plasma Physics and Controlled Fusion (Ple-
} num, New York, 1984). 